\title{
DEGRADAÇÃO ELETROQUÍMICA DO CLORANFENICOL EM REATOR DE FLUXO
}

\section{Luis Gustavo P. Rezende e Vânia M. do Prado}

Universidade São Francisco, Campus de Bragança Paulista, 12916-900 Bragança Paulista - SP, Brasil

Robson S. Rocha

Departamento de Engenharia de Materiais, Faculdade de Engenharia Mecânica, Universidade Estadual de Campinas, 13083-970 Campinas - SP, Brasil

André A. G. F. Beati

Universidade São Francisco, Campus de Bragança Paulista, 12.16-900 Bragança Paulista - SP / Departamento de Engenharia de Materiais, Faculdade de Engenharia Mecânica, Universidade Estadual de Campinas, 13083-970 Campinas - SP, Brasil

Maria Del Pilar T. Sotomayor

Departamento de Química Analítica, Instituto de Química de Araraquara, Universidade Estadual Paulista, 01049-010 Araraquara - SP, Brasil

Marcos R. V. Lanza*

Departamento de Química e Física Molecular, Instituto de Química de São Carlos, Universidade de São Paulo, 13560-970 São Carlos - SP, Brasil

Recebido em 19/8/09; aceito em 18/1/10; publicado na web em 23/4/10

\begin{abstract}
ELECTROCHEMICAL DEGRADATION OF THE CHLORAMPHENICOL AT FLOW REACTOR. This paper reports a study of electrochemical degradation of the chloramphenicol antibiotic in aqueous medium using a flow-by reactor with DSA ${ }^{\circledR}$ anode. The process efficiency was monitored by chloramphenicol concentration analysis with liquid chromatography (HPLC) during the experiments. Analysis of Total Organic Carbon (TOC) was performed to estimate the degradation degree and Ion Chromatography (IC) was performed to determinate inorganic ions formed during the eletrochemical degradation process. In electrochemical flow-by reactor, $52 \%$ of chloramphenicol was degraded, with $12 \%$ TOC reduction. IC analysis showed the production of chloride ions (25 $\left.\mathrm{mg} \mathrm{L}^{-1}\right)$, nitrate ions $\left(6 \mathrm{mg} \mathrm{L}^{-1}\right)$ and nitrite ions $\left(4.5 \mathrm{mg} \mathrm{L}^{-1}\right)$.
\end{abstract}

Keywords: chloramphenicol; electrochemical reactor; DSA ${ }^{\circledR}$ anodes.

\section{INTRODUÇÃO}

Atualmente, diversas substâncias têm despertado interesse no que concerne ao seu impacto ambiental, principalmente em ambientes aquáticos, principalmente devido às elevadas estabilidades e toxicidades. No caso de substâncias biologicamente ativas, o sistema de tratamento convencional das águas de abastecimento pode não ser totalmente eficaz para a degradação destas substâncias, o que torna vital o tratamento de efluentes contendo estas substâncias, evitando a contaminação dos rios e lagos e colocando em risco o meio ambiente como um todo.

Diversas classes de medicamentos como antibióticos, hormônios, anti-inflamatórios dentre outros, já foram detectados em esgoto doméstico, em águas superficiais e de subsolo. O uso desenfreado destas substâncias acarreta dois sérios problemas ambientais: a contaminação dos recursos hídricos e a possível mutação de micro-organismos. ${ }^{1,2}$

Os antibióticos são uma classe de medicamentos que têm sido amplamente discutidos na literatura por serem comercializados em grandes quantidades e dentre os estes está o cloranfenicol, um antibiótico conhecido pelo seu amplo espectro antibacteriano, com utilização na medicina humana e animal, tanto pela sua eficácia, quanto pelo seu baixo custo. Por ser um antibiótico muito empregado, existe a preocupação em monitorar a presença deste medicamento em águas residuais em vários países e propor possíveis processos de tratamento para este fármaco, antes descartar a água residuária na natureza. ${ }^{1,3-6}$

O grande consumo dos medicamentos contendo cloranfenicol, sua diversificada forma de apresentação e sua variada fonte de geração de resíduos dentro da indústria, torna o estudo da degradação desse

\footnotetext{
*e-mail: marcoslanza@iqsc.usp.br
}

composto ativo e o consequente tratamento das águas residuárias da industria farmacêutica de grande importância, devido ao impacto que esses compostos biologicamente ativos causam no meio ambiente. Para o controle desses compostos ativos existentes nos despejos da indústria farmacêutica, existem vários processos de tratamento de efluentes aquosos que podem ser utilizados, como o tratamento químico e a incineração do efluente.

Estes processos de tratamento usualmente utilizados na indústria apresentam algumas desvantagens, como o aumento do volume do efluente no tratamento químico ou geração de outras classes de resíduos na incineração. Diante das limitações dos tratamentos usuais de compostos da indústria farmacêutica, abrem-se precedentes para a utilização de técnicas não convencionais, como as técnicas eletroquímicas para o tratamento de efluentes. ${ }^{7}$

Os tratamentos eletroquímicos de efluentes promovem a remoção ou a destruição de espécies poluentes, direta ou indireta, através de processos eletroquímicos de oxidação e/ou redução em células eletroquímicas, sem a adição de grandes quantidades de produtos químicos evitando, assim, possível formação de organometálicos e o aumento do volume a ser tratado.

Nesse contexto, a tecnologia eletroquímica oferece um meio eficiente de controle da poluição por meio de reações redox diretas entre as espécies poluentes e as superfícies eletródicas contendo certos metais/óxidos, comumente indicados na literatura como eficientes na degradação de compostos orgânicos..$^{8-11}$

Os eletrodos de titânio revestidos com óxidos, baseados em $\mathrm{RuO}_{2}$, $\mathrm{IrO}_{2}, \mathrm{TiO}_{2} / \mathrm{RuO}_{2}, \mathrm{Ta}_{2} \mathrm{O}_{5} / \mathrm{IrO}_{2}$, foram popularizados na literatura. ${ }^{8-12} \mathrm{Es}-$ tes revestimentos, principalmente $\mathrm{TiO}_{2} / \mathrm{RuO}_{2}$, aplicados sobre placas de titânio são comercializados como DSA ${ }^{\circledR}$ (Dimensionally Stable 
Anodes). O titânio é usado como substrato pelas suas características de resistência à corrosão e pela sua capacidade de passivação em caso de falhas do revestimento, mesmo em potenciais muito positivos. ${ }^{11,13}$

A utilização dos eletrodos de óxidos de titânio e rutênio $\left(\mathrm{TiO}_{2} /\right.$ $\mathrm{RuO}_{2}$ ) tem o objetivo de se beneficiar de uma característica única deste tipo de eletrodo que é a adsorção de espécies atômicas e radicalares, intermediárias da reação de desprendimento de oxigênio. ${ }^{11} \mathrm{~A}$ descarga anódica da água sobre o eletrodo de óxido $\left(\mathrm{MO}_{\mathrm{x}}\right)$ tem início através da formação de radicais hidroxila $\left({ }^{\bullet} \mathrm{OH}\right)$ que adsorvem fisicamente na superfície, de acordo com a Equação 1:11,13

$$
\mathrm{MO}_{\mathrm{x}}+\mathrm{H}_{2} \mathrm{O} \rightarrow \mathrm{MO}_{\mathrm{x}}\left(\bullet^{\circ} \mathrm{OH}\right)+\mathrm{H}^{+}+\mathrm{e}^{-}
$$

Conforme descrito por Simond et al., a adsorção do radical hidroxila ocorre como uma espécie intermediária na formação de óxidos superiores $\left(\mathrm{MO}_{\mathrm{x}+1}\right)$, conforme a Equação 2.

$$
\mathrm{MO}_{\mathrm{x}}(\bullet \mathrm{OH}) \rightarrow \mathrm{MO}_{\mathrm{x}+1}+\mathrm{H}^{+}+\mathrm{e}^{-}
$$

Segundo os autores, a espécie $\mathrm{MO}_{\mathrm{x}+1}$ é responsável pela oxidação de compostos orgânicos, como descrito na Equação 3, porém, estes óxidos superiores também são responsáveis pela reação de desprendimento de oxigênio no eletrodo, Equação 4, reação esta que pode interferir negativamente na eficiência da oxidação do composto orgânico, pois utiliza a espécie $\mathrm{MO}_{\mathrm{x}+1}$ para a formação de $\mathrm{O}_{2}$, conforme descrito na Equação 4. ${ }^{11,13}$

$$
\begin{gathered}
\mathrm{MO}_{\mathrm{x}+1}+\mathrm{R} \rightarrow \mathrm{MO}_{\mathrm{x}}+\mathrm{RO} \\
\mathrm{MO}_{\mathrm{x}+1} \rightarrow \mathrm{MO}_{\mathrm{x}}+1 / 2 \mathrm{O}_{2}
\end{gathered}
$$

Neste trabalho são apresentados os resultados da degradação eletroquímica de efluentes simulados da indústria farmacêutica contendo cloranfenicol, utilizando um reator eletroquímico de fluxo com um anodo do tipo DSA ${ }^{\circledR}$.

\section{PARTE EXPERIMENTAL}

O estudo da degradação eletroquímica do cloranfenicol foi realizado em um reator eletroquímico de bancada, utilizando soluções contendo o fármaco. Foi avaliada a influência das principais condições operacionais na degradação do cloranfenicol.

\section{Reator eletroquímico}

Para a realização das eletrólises foi utilizado um reator eletroquímico do tipo flow-by com um compartimento, construído com placas de PVC montadas na forma de um filtro-prensa, projetado para ser acoplado em um reservatório com capacidade máxima de 2,8 L. ${ }^{14,15}$

O reator utilizou um eletrodo do tipo EDG com $20 \%$ de politetrafluoretileno (PTFE) na placa catódica (com uma área geométrica de $\approx 20 \mathrm{~cm}^{2}$ ), atuando como catodo de carbono, e um anodo comercial DSA-Cl ${ }_{2}^{\circledR}$ da De Nora do Brasil Ltda (com uma área geométrica de $20 \mathrm{~cm}^{2}$ ). Entre as duas placas, anódica e catódica, foi colocado um espaçador, estabelecendo uma distância de $3 \mathrm{~mm}$. O reator foi conectado a um sistema de recirculação, composto por um reservatório, uma bomba hidráulica e um medidor de vazão do tipo rotâmetro. Em todos os ensaios, o sistema do reator operou no modo de recirculação.

\section{Degradação do cloranfenicol}

Para o estudo da degradação do cloranfenicol foi utilizada a técnica de eletrólise a corrente constante a partir do acoplamento de uma fonte de tensão estabilizada Tectrol TC 20-05 ao sistema do reator eletroquímico. Para a realização das eletrólises foram estabelecidas as densidades de corrente anódica de 50,100, 150, 200 e $250 \mathrm{~mA}$

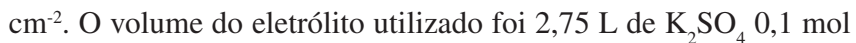
$\mathrm{L}^{-1}+\mathrm{H}_{2} \mathrm{SO}_{4} 0,1 \mathrm{~mol} \mathrm{~L}^{-1}$ com $200 \mathrm{mg} \mathrm{L}^{-1}$ de cloranfenicol em vazões de 50 e $350 \mathrm{~L} \mathrm{~h}^{-1}$ e em todos os ensaios foram aplicados fluxo de $\mathrm{N}_{2}$ no EDG com pressão de 0,2 Bar. Durante os experimentos foram retiradas amostras (aproximadamente $15 \mathrm{~mL}$ ) nos tempos $0,5,10$, 20, 30, 60 e $120 \mathrm{~min}$.

\section{Instrumentação e procedimentos de análise}

As análises da concentração de cloranfenicol em função do tempo de eletrólise foram realizadas em um cromatógrafo líquido de alta eficiência (CLAE) da Shimadzu modelo 20A, com detector UV/Visível SPD-20A. A fase estacionária utilizada foi uma coluna 250 x 4,6 mm (coluna $\mathrm{C}_{18}$ ) e a fase móvel composta de uma mistura de água, metanol e ácido acético em uma proporção de 55:45:0,1, com fluxo de 1,0 $\mathrm{mL} \mathrm{min}^{-1}$, o comprimento de onda analisado pelo detector UV/Vis foi $280 \mathrm{~nm}$ e a temperatura do forno foi de $35^{\circ} \mathrm{C} .{ }^{16}$ Para a detecção e quantificação do íons inorgânicos formados durante a degradação foi utilizada cromatografia de íons (IC) em um cromatógrafo de íons modelo Pro 850 da Metrohm, com detector de condutividade, coluna de ânions A Supp 5 com $150 \mathrm{~mm}$, o eluente utilizado foi uma solução de carbonato de sódio $3,2 \times 10^{-3} \mathrm{~mol} \mathrm{~L}^{-1} \mathrm{e}$ bicarbonato de sódio $1,0 \times 10^{-3} \mathrm{~mol} \mathrm{~L}^{-1}$, com fluxo de $0,7 \mathrm{~mL} \mathrm{~min}^{-1} \mathrm{e}$ volume de injeção de $20 \mu \mathrm{L}$ de amostra. Um analisador de carbono orgânico total modelo TOC-VCPN da Shimadzu foi empregado na determinação dos teores de carbono orgânico total (COT) durante os ensaios eletroquímicos.

\section{RESULTADOS E DISCUSSÃO}

O processo de degradação do cloranfenicol em reator eletroquímico de fluxo baseia-se na reação de oxidação do fármaco na superfície do anodo (DSA ${ }^{\circledR}$ ), sendo que o uso do EDG como catodo teve como objetivo compor o circuito elétrico no reator, sem interferir diretamente no processo de degradação da matéria orgânica presente no eletrólito. Com o intuito de evitar a formação de $\mathrm{H}_{2} \mathrm{O}_{2}$ a partir da reação de $\mathrm{O}_{2}$ no catodo, empregando-se um eletrodo de difusão gasosa, aplicou-se fluxo positivo de $\mathrm{N}_{2}$ através do EDG, pela imposição de 0,2 Bar de pressão na câmara externa do EDG. ${ }^{17,18}$

Considerando-se o regime hidrodinâmico no interior do reator, utilizaram-se as vazões de 50 e $350 \mathrm{~L} \mathrm{~h}^{-1}$ para avaliar a eficiência do processo de degradação eletroquímica, as quais apresentam, respectivamente, regime laminar (Número de Reynolds; $\mathrm{Re} \approx 500$ ) e regime turbulento (Número de Reynolds; $R e \approx 4500$ ). Os experimentos a corrente constante foram realizados em cada uma das vazões e os decaimentos da concentração normalizada do cloranfenicol em função do tempo de ensaio, utilizando-se a CLAE para as duas vazões utilizadas, são apresentados na Figura 1.

Nas Figuras 1A e 1B, observa-se que a concentração normalizada do cloranfenicol apresenta um decaimento linear em função do tempo de eletrólise, com uma expressiva redução na concentração do fármaco em função do tempo de ensaio com o aumento da densidade de corrente aplicada, independente da vazão estudada. Na Figura 1A (50 $\mathrm{L} \mathrm{h}^{-1}$ ), a redução da concentração de cloranfenicol variou de 18 a $40 \%$ nas densidades de corrente de 50 a $250 \mathrm{~mA} \mathrm{~cm}^{-2}$, respectivamente. $\mathrm{Na}$ Figura 1B $\left(350 \mathrm{~L} \mathrm{~h}^{-1}\right)$, nota-se uma maior eficiência no processo de degradação do cloranfenicol, quando comparada aos mesmos valores de densidade de corrente aplicada na vazão, com uma remoção variando de 33 a $52 \%$ da concentração inicial do composto orgânico aplicando-se 50 e $250 \mathrm{~mA} \mathrm{~cm}^{-2}$, respectivamente. 

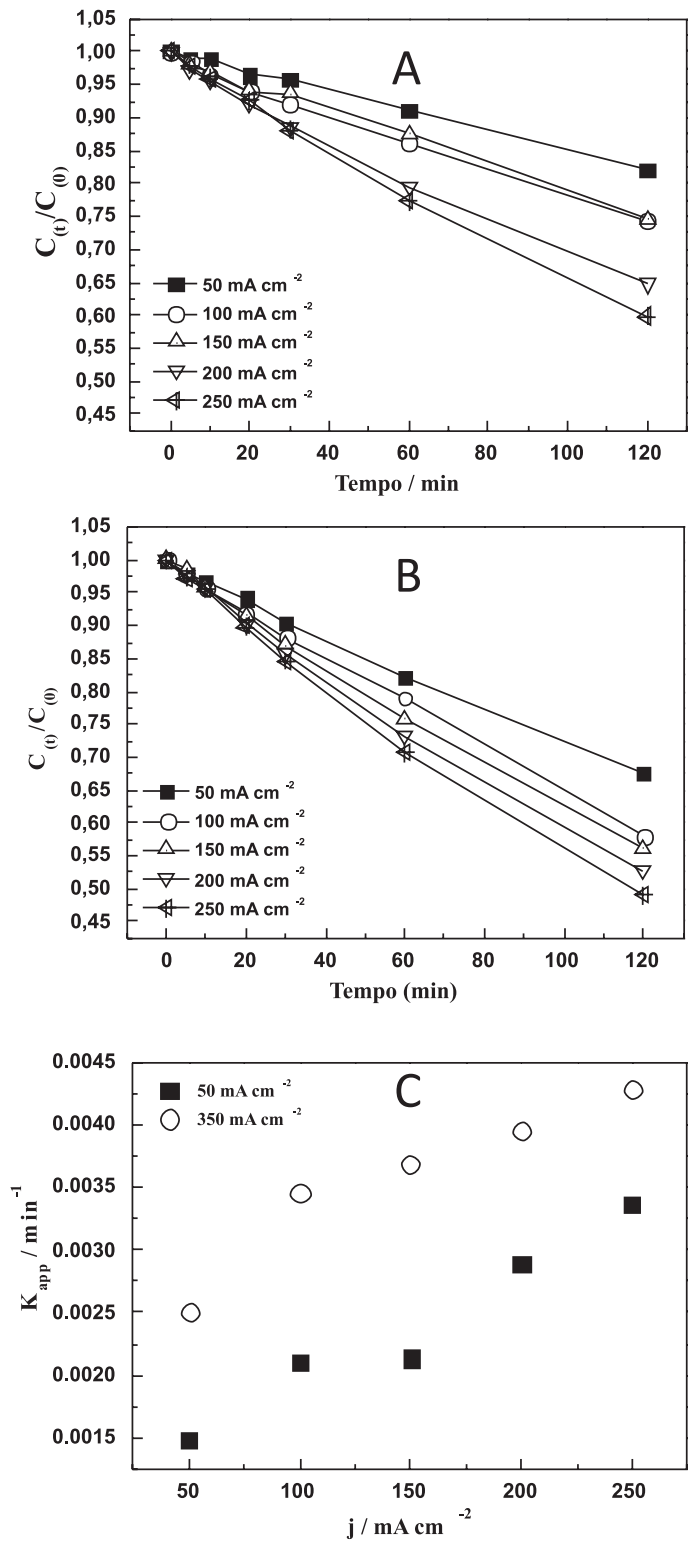

Figura 1. Decaimento da concentração normalizada de diclofenaco obtido pela técnica de CLAE, em função do tempo de eletrólise, nas densidades de corrente mostradas na figura. Eletrólito: $2,75 \mathrm{~L}_{\text {de }} \mathrm{K}_{2} \mathrm{SO}_{4}$ 0,1 $\mathrm{mol} \mathrm{L}^{-1}+$ $\mathrm{H}_{2} \mathrm{SO}_{4} 0,1 \mathrm{~mol} \mathrm{~L}^{-1} \mathrm{com} \approx 200 \mathrm{mg} \mathrm{L}^{-1}$ de cloranfenicol. (A) Vazão do reator em $50 \mathrm{~L} \mathrm{~h}^{-1}$; (B) vazão do reator em $350 \mathrm{~L} \mathrm{~h}^{-1}$; (C) constante aparente global do decaimento da concentração do cloranfenicol nas vazões de 50 e $350 \mathrm{~L} \mathrm{~h}^{-1}$

Nas Figuras 1A e 1B, nota-se o mesmo comportamento linear para a variação na concentração normalizada do fármaco em função do tempo nas duas vazões estudadas. Apesar de se esperar um comportamento exponencial do decaimento da concentração normalizada do cloranfenicol, característico de uma cinética de primeira ordem, o decaimento linear apresentado pode ser decorrente da correlação entre a área anódica do reator (área geométrica de aproximadamente $20 \mathrm{~cm}^{2}$ ) e o volume de eletrólito contendo uma concentração considerável do cloranfenicol $\left(\approx 2,75 \mathrm{~L}\right.$ contendo aproximadamente $\left.200 \mathrm{mg} \mathrm{L}^{-1}\right)$, além da elevada estabilidade da molécula, o que, nas condições dos ensaios, induziu a um comportamento cinético mais lento de degradação, característico de uma cinética de pseudo-ordem zero. Este comportamento pode indicar, ainda, que o sistema do reator eletroquímico está operando em valores de densidade de corrente abaixo da corrente limite que promoveria um processo cinético de primeira ordem.
Neste caso, calculou-se uma constante cinética aparente $\left(\mathrm{K}_{\text {app }}\right)$ a partir do coeficiente angular das curvas de decaimento normalizado das concentrações de cloranfenicol em função do tempo de eletrólise, assumindo-se uma cinética de pseudo-ordem zero. Na Figura 1C, são mostrados os valores de $\mathrm{K}_{\text {app }}$ para cada uma das densidades de corrente aplicadas nas duas vazões de trabalho. Nota-se que os valores das constantes aparentes aumentam com o aumento da corrente aplicada e que, na comparação entre as vazões aplicadas, os experimentos a $350 \mathrm{~L} \mathrm{~h}^{-1}$ apresentaram valores superiores se comparados aos valores apresentados nos experimentos a $50 \mathrm{~L} \mathrm{~h}^{-1}$.

Este comportamento é um indicativo de que em condições hidrodinâmicas que tendem a promover a reposição das moléculas do composto orgânico na superfície do eletrodo com uma maior taxa, com a diminuição da camada de difusão e o favorecimento do processo de oxidação via transferência eletrônica entre a superfície anódica e/ou espécies oxidantes quimicamente adsorvidas e as moléculas do fármaco, promoveriam uma alteração da cinética do sistema de pseudo-ordem zero para primeira ordem (aumento no valor do Número de Reynolds).

Os resultados das análises via CLAE mostraram que a diminuição da concentração do cloranfenicol está associada à densidade de corrente aplicada, provavelmente devido a maior formação de espécies oxidantes na superfície do eletrodo, como descrito na Equação 2. Outro fator observado foi a maior redução na concentração do fármaco quando se realizaram os experimentos a $350 \mathrm{~L} \mathrm{~h}^{-1}$ se comparados aos experimentos a $50 \mathrm{~L} \mathrm{~h}^{-1}$; possivelmente, a maior redução em $350 \mathrm{~L}$ $\mathrm{h}^{-1}$ se deve ao regime hidrodinâmico do reator nesta vazão, pois no regime turbulento ocorre uma maior remoção das espécies oxidadas da superfície do eletrodo e, também, uma melhor reposição de novas espécies na interface eletrodo/eletrólito melhorando, assim, a oxidação das moléculas do cloranfenicol na superfície do DSA ${ }^{\circledR}$.

Observando os resultados da Figura 1 nota-se a diminuição da concentração do cloranfenicol em até aproximadamente 50\%. Esses resultados são inferiores se comparados com outros trabalhos da literatura que utilizam o mesmo reator eletroquímico do tipo flow-by, ${ }^{14,15}$ porém, nestes trabalhos os processos de degradação foram baseados na eletrogeração de $\mathrm{H}_{2} \mathrm{O}_{2}$ e na utilização de agente oxidante para promover a degradação dos compostos orgânnicos via um processo eletro-Fenton (adição de íons Fe(II)).

Como não é possível, através da cromatografia líquida, determinar a mineralização da carga orgânica presente no eletrólito, utilizou-se a análise do teor de carbono orgânico total (COT) ao final dos ensaios, sendo que os resultados são apresentados na Tabela 1. Observando os resultados finais de remoção de COT, nota-se que a densidade de corrente aplicada influencia diretamente a taxa de remoção de carbono orgânico, onde a menor densidade de corrente removeu 8 e $6 \%$ em 50 e $350 \mathrm{~L} \mathrm{~h}^{-1}$, respectivamente, e a maior densidade de corrente utilizada removeu as maiores quantidades de carbono orgânico com $12 \%$ de remoção para as duas vazões utilizadas.

Tabela 1. Remoção, em \%, do COT ao final dos experimentos de $2 \mathrm{~h}$ em função das correntes aplicadas, nas vazões de 50 e $350 \mathrm{~L} \mathrm{~h}^{-1}$

\begin{tabular}{ccc}
\hline $\mathrm{i} / \mathrm{mA} \mathrm{cm} \mathrm{c}^{-2}$ & $50 \mathrm{~L} \mathrm{~h}^{-1}$ & $350 \mathrm{~L} \mathrm{~h}^{-1}$ \\
\hline 50 & $8,0 \%$ & $6,0 \%$ \\
100 & $9,3 \%$ & $6,4 \%$ \\
150 & $9,6 \%$ & $7,6 \%$ \\
200 & $9,8 \%$ & $8,2 \%$ \\
250 & $12,0 \%$ & $12,0 \%$ \\
\hline
\end{tabular}

A variação da vazão do eletrólito no reator não influenciou na remoção do carbono orgânico total, sendo que nos experimentos com vazão a $50 \mathrm{~L} \mathrm{~h}^{-1}$ foram obtidas remoções de COT de 8 a $12 \%$ (50 e 250 
$\mathrm{mA} \mathrm{cm}{ }^{-2}$, respectivamente) e nos experimentos a $350 \mathrm{~L} \mathrm{~h}^{-1}$, de 6 a $12 \%$ (50 e $250 \mathrm{~mA} \mathrm{~cm}^{-2}$, respectivamente). Este comportamento é um forte indicativo da estabilidade da molécula do cloranfenicol à degradação induzida pelo processo eletroquímico direto, pois a diminuição das concentrações do fármaco observadas nas análises cromatográficas, sem a correspondente mineralização, estaria associada à formação de intermediários de degradação com absorção em comprimentos de onda diferentes do utilizado para a detecção do composto.

Com a diminuição da concentração do cloranfenicol monitorada pelo CLAE (Figura 1) em torno de $50 \%$ e a redução da concentração de COT em torno de $12 \%$ (Tabela 2), pode-se avaliar que ocorreu a quebra da molécula do cloranfenicol ao invés da mineralização da carga orgânica; esse fato pode ter contribuído para a formação de subprodutos da reação da degradação do fármaco e uma das possibilidades de subprodutos é a formação de íons inorgânicos originados durante o processo, a partir dos átomos de cloro e nitrogênio presentes na molécula do cloranfenicol. Neste caso, utilizou-se a cromatografia de íons (IC) para a identificação e quantificação dos possíveis íons inorgânicos formados nos processos de degradação eletroquímica. As análises de IC mostraram a formação de íons cloreto, nitrito e nitrato, sendo que as concentrações máximas dos íons inorgânicos formados em função do tempo de eletrólise nas diferentes vazões estudadas são apresentadas na Tabela 2.

Tabela 2. Concentração máxima $\left(\mathrm{mg} \mathrm{L}^{-1}\right)$ dos íons cloreto, nitrato e nitrito em função do tempo de eletrólise, nas densidades de corrente de estudo. Eletrólito: $2,75 \mathrm{~L}_{\text {de }} \mathrm{K}_{2} \mathrm{SO}_{4} 0,1 \mathrm{~mol} \mathrm{~L}^{-1}+\mathrm{H}_{2} \mathrm{SO}_{4} 0,1 \mathrm{~mol} \mathrm{~L}^{-1}$ com $200 \mathrm{mg} \mathrm{L}^{-1}$ de cloranfenicol. Vazão do reator em 50 e $350 \mathrm{~L} \mathrm{~h}^{-1}$.

\begin{tabular}{ccccccc}
\hline & \multicolumn{3}{c}{$50 \mathrm{~L} \mathrm{~h}^{-1}$} & \multicolumn{3}{c}{$350 \mathrm{~L} \mathrm{~h}^{-1}$} \\
$\mathrm{i} / \mathrm{mA} \mathrm{cm}^{-2}$ & $\mathrm{Cl}^{-}$ & $\mathrm{NO}_{3}^{-}$ & $\mathrm{NO}_{2}^{-}$ & $\mathrm{Cl}^{-}$ & $\mathrm{NO}_{3}^{-}$ & $\mathrm{NO}_{2}^{-}$ \\
\hline 50 & 8,3 & 4,1 & 0,0 & 8,9 & 4,1 & 0,0 \\
100 & 15,4 & 6,2 & 0,0 & 12,2 & 6,6 & 0,0 \\
150 & 16,3 & 7,6 & 0,0 & 16,3 & 7,5 & 0,0 \\
200 & 23,2 & 6,3 & 4,6 & 18,1 & 6,3 & 4,6 \\
250 & 25,5 & 6,1 & 4,6 & 21,8 & 5,9 & 0,0 \\
\hline
\end{tabular}

Na Tabela 2, podem-se observar as concentrações dos íons inorgânicos em diferentes densidades de corrente aplicadas nas vazões estudadas. Nota-se que a formação de íons cloreto ocorre em todas as correntes utilizadas e nas duas vazões de estudo, a concentração máxima do íon cloreto aumenta com o aumento da densidade de corrente aplicada alcançando valores máximos em $250 \mathrm{~mA} \mathrm{~cm}^{-2}$, com 25,5 e 21,8 $\mathrm{mg} \mathrm{L}^{-1}$ de íons cloreto em 50 e $350 \mathrm{~L} \mathrm{~h}^{-1}$, respectivamente, indicando um processo intensivo de dealogenação decorrente do processo de oxidação eletroquímica.

Em relação aos íons nitrato, ocorre o aumento na formação dos íons com o aumento da densidade de corrente aplicada até $150 \mathrm{~mA}$ $\mathrm{cm}^{-2}$ com 7,6 $\mathrm{mg} \mathrm{L}^{-1}$ de íons nitrato nas duas vazões estudadas, porém nas densidades de corrente superiores se observou uma diminuição da concentração, alcançando valores finais de $6 \mathrm{mg} \mathrm{L}^{-1} \mathrm{em} 250 \mathrm{~mA}$ $\mathrm{cm}^{-2}$ nas duas vazões. Observando-se a concentração dos íons nitrito, nota-se que o íon não foi detectado em todos os experimentos. $\mathrm{Na}$ vazão $50 \mathrm{~L} \mathrm{~h}^{-1}$ a formação do íon ocorreu apenas nas eletrólises a 200 e $250 \mathrm{~mA} \mathrm{~cm}^{-2}$, alcançando $4,6 \mathrm{mg} \mathrm{L}^{-1}$. No experimento a $200 \mathrm{~mA}$ $\mathrm{cm}^{-2}$ a formação do íon nitrito ocorreu durante todo o experimento mantendo concentrações muito próximas a $4,6 \mathrm{mg} \mathrm{L}^{-1}$, entretanto, no experimento a $250 \mathrm{~mA} \mathrm{~cm}{ }^{-2}$ a detecção do íon ocorreu somente até $30 \mathrm{~min}$ de experimento, não sendo mais detectado até o final do experimento. Na vazão de $350 \mathrm{~L} \mathrm{~h}^{-1}$, a detecção do íon nitrito ocorreu somente no experimento a $200 \mathrm{~mA} \mathrm{~cm}^{-2}$, onde a concentração alcançou valores próximos a $4,6 \mathrm{mg} \mathrm{L}^{-1}$ durante todo o experimento.
A análise dos resultados sugere que a formação dos íons inorgânicos, durante os experimentos de degradação do cloranfenicol, não segue a mesma rota, pois a formação dos íons cloreto aumenta com o aumento da densidade de corrente aplicada, da mesma maneira que o íon nitrato apresenta variação na concentração semelhante ao cloreto, porém o aumento é observado até $150 \mathrm{~mA} \mathrm{~cm}^{-2}$ com posterior diminuição da concentração em função do aumento da densidade de corrente e a detecção do íon nitrito na eletrólise a $200 \mathrm{~mA} \mathrm{~cm}{ }^{-2}$, durante todo o experimento.

Essa variação na concentração dos íons durante as eletrólises pode estar associada ao tipo de clivagem que ocorre na molécula do cloranfenicol, como é apresentado na Figura 2. Nesta Figura, podemse observar as regiões de formação dos íons inorgânicos na molécula do cloranfenicol. A formação dos íons cloretos pode ser explicada pela facilidade de remoção dos átomos de cloro da região A, devido à menor estabilidade desta região da molécula. ${ }^{19}$ Esse fator pode ser observado também na região $\mathrm{B}$, onde possivelmente ocorre a formação do íon nitrato. Com os resultados nota-se que a formação destes íons também se beneficia da estrutura da molécula do cloranfenicol, proferindo uma relativa facilidade na formação dos íons nitrato, porém este íon alcança concentrações finais menores se comparado com o íon cloreto. Com relação à formação do íon nitrito, região C, nota-se a complexidade para a liberação do nitrogênio desta região, devido à exigência da quebra de algumas ligações para liberar o respectivo átomo; essa maior dificuldade pode ser explicada pelo íon nitrito só ser detectado nas amostras do experimento com $200 \mathrm{~mA} \mathrm{~cm}^{-2}$.

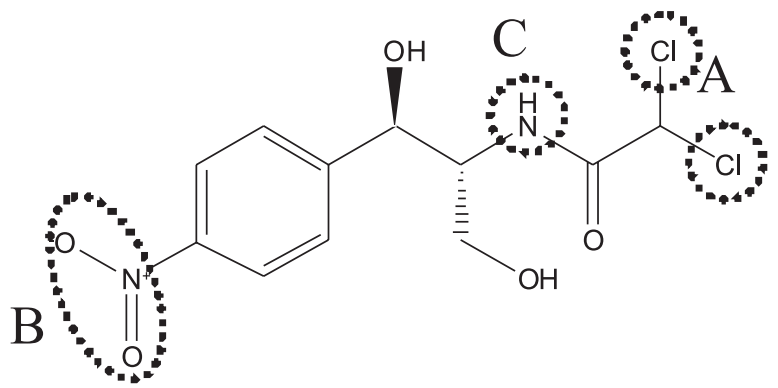

Figura 2. Fórmula estrutural do cloranfenicol com as possíveis regiões de formação dos íons inorgânicos

Com os resultados da formação dos íons inorgânicos, pode-se notar que a maior formação dos íons cloreto e nitrato pode estar associada à localização nas extremidades da molécula das regiões A e B e que o íon nitrito apresentou concentrações menores e em uma só densidade de corrente, devido à localização no centro da molécula, dificultando assim a liberação do átomo de nitrogênio da região $\mathrm{C}$.

Diante dos resultados do decaimento da concentração do cloranfenicol em até $50 \%$, da diminuição da carga de carbono orgânico em até $12 \%$ e a formação dos íons inorgânicos nas densidades de corrente, pode-se considerar que ocorreu a diminuição da concentração do cloranfenicol pela clivagem da sua estrutura e consequente formação dos íons inorgânicos, porém a pequena diminuição do COT (12\%) indica uma baixa taxa de mineralização das amostras. Com a análise dos resultados pode-se propor possíveis subprodutos derivados somente da clivagem da molécula e formação dos íons inorgânicos, como descrito na Figura 3.

Na Figura 3 é esquematizada uma proposta de possíveis rotas de degradação e com possíveis subprodutos formados a partir da degradação do cloranfenicol. Esta proposta está baseada na formação dos íons inorgânicos durante as eletrólises a corrente constante nas vazões de 50 e $350 \mathrm{~L} \mathrm{~h}^{-1}$. Observando a clivagem da molécula do fármaco, nota-se a tendência geral de formação de dois subprodutos 


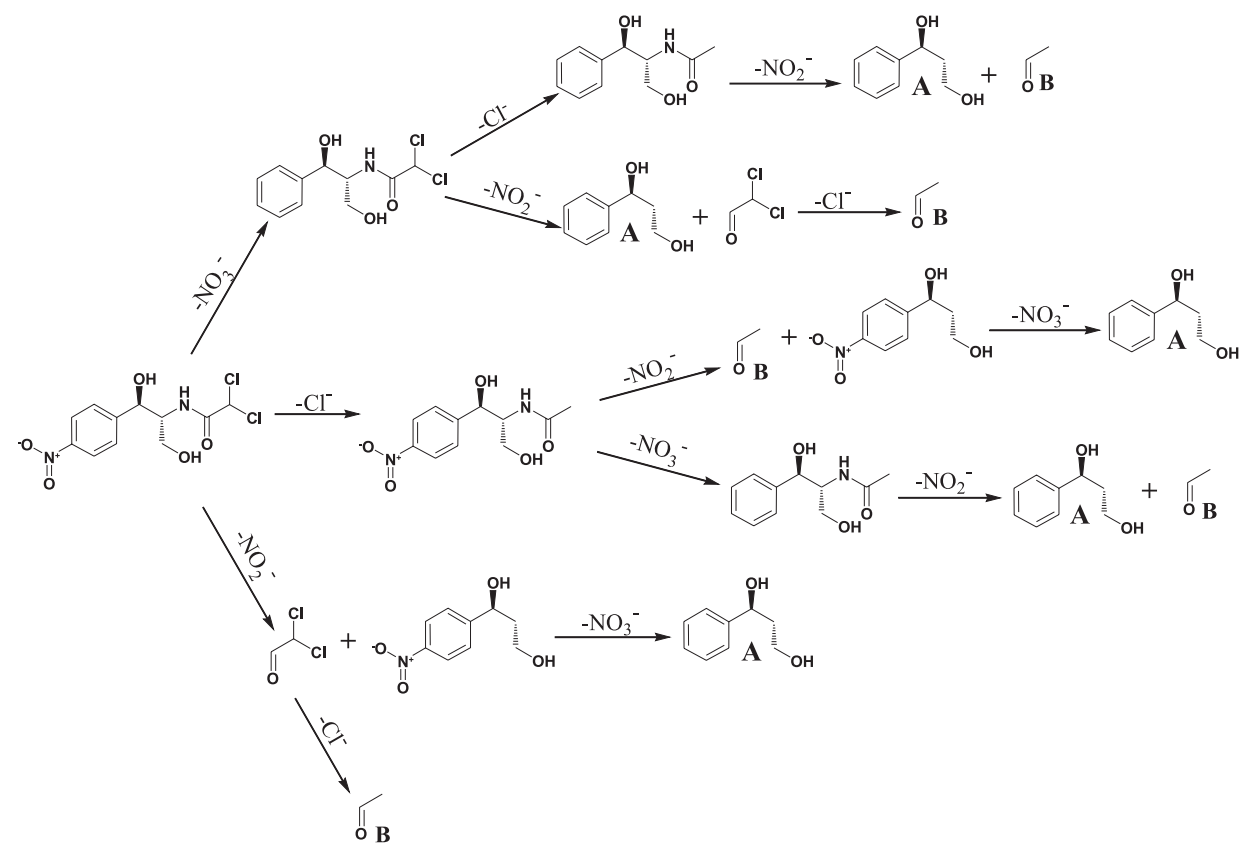

Figura 3. Rota de formação dos possiveis sub-produtos derivados da formação dos íons inorgânicos

orgânicos (compostos A e B da Figura 3), independente da rota de degradação, com a formação dos íons inorgânicos cloreto, nitrato e nitrito a partir dos heteroátomos presentes na molécula. No entanto, para uma confirmação da formação dos subprodutos e a sua eventual identificação (e confirmação), seria necessária a utilização de uma técnica de elucidação estrutural como a cromatografia líquida associada à espectrometria de massas.

\section{CONCLUSÃO}

A degradação do cloranfenicol em um reator eletroquímico do tipo flow by apresentou resultados promissores. A avaliação dos resultados mostrou que os experimentos nas vazões de $50 \mathrm{~L} \mathrm{~h}^{-1}$ (regime hidrodinâmico laminar) e $350 \mathrm{~L} \mathrm{~h}^{-1}$ (regime hidrodinâmico turbulento) apresentaram resultados semelhantes.

No monitoramento da concentração do cloranfenicol via CLAE, o aumento de densidade de corrente promoveu uma redução máxima de aproximadamente $40 \%$ na vazão de $50 \mathrm{~L} \mathrm{~h}^{-1}$ e $52 \%$ na vazão de $350 \mathrm{~L} \mathrm{~h}^{-1}$. Essa tendência se repetiu quando foram analisadas as concentração de carbono orgânico total, onde os experimentos nas duas vazões estudadas apresentaram resultados próximos, $12 \%$ de remoção de COT.

Com relação aos produtos formados na degradação do cloranfenicol, observou-se a formação dos íons cloreto, em $25,5 \mathrm{mg} \mathrm{L}^{-1}$ a $50 \mathrm{~L} \mathrm{~h}^{-1}$ e $21,8 \mathrm{mg} \mathrm{L}^{-1}$ a $350 \mathrm{~L} \mathrm{~h}^{-1}$; formação dos íons nitrato, em 6,1 $\mathrm{mg} \mathrm{L}^{-1}$ nas duas vazões e os íons nitrito, aproximadamente em 4,6 $\mathrm{mg} \mathrm{L}^{-1}$ nas duas vazões. A partir destas informações inferiu-se sobre as possíveis rotas de degradação com a indicação de subprodutos orgânicos e inorgânicos.

\section{AGRADECIMENTOS}

À Fundação de Amparo à Pesquisa do Estado de São Paulo FAPESP.

\section{REFERÊNCIAS}

1. Bila, D. M.; Desotti, M.; Quim. Nova 2003, 26, 523.

2. Mispagel, H.; Gray, J. T.; Water Environ. Res. 2005, 77, 2996.

3. Peng, X.; Wang, Z.; Kuang, W.; Tan, J.; Li, K.; Sci. Total Environ. 2006, $371,314$.

4. Peng, X.; Tan, J.; Tang, C.; Yu, Y.; Wang, Z.; Environ. Toxicol. Chem. 2008, 27, 73 .

5. Zeegers, F.; Gibella, M.; Tilquin, B.; Radiat. Phys. Chem. 1997, 50, 149.

6. Chatzitakis, A.; Berberidou, C.; Paspaltsis, I.; Kyriakou, G.; Sklaviadis, T.; Poulios, I.; Water Res. 2008, 42, 386.

7. Di Bernardo, L.; Dantas, A. D. B.; Métodos e Técnicas de Tratamento de Água, $2^{\mathrm{a}}$ ed., Rima: São Paulo, 2005.

8. Faria, L. A.; Boodts, J. F. C.; Trassati, S.; Electrochim. Acta 1992, 37, 2511.

9. Comninellis, Ch.; Sequeira, C. A. C.; Environmental Oriented Electrochemistry 1994, 77.

10. Forti, G.; Gandini, D.; Comninellis, Ch.; Curr. Top. Electrochem. 1997, 5,71 .

11. Simond, O.; Schaller, V.; Comninellis, Ch.; Electrochim. Acta 1997, 42, 2009.

12. Trassati, S.; Electrochim. Acta 2000, 45, 2377.

13. Comninellis, Ch.; Electrochim. Acta 1994, 39, 1857.

14. Rocha, R. S.; Beati, A. A. G. F.; Oliveira, J. G.; Lanza, M. R. V.; Quim. Nova 2009, 32, 354.

15. Beati, A. A. G. F.; Rocha, R. S.; Oliveira, J. G.; Lanza, M. R. V.; Quim. Nova 2009, 32, 125.

16. The Pharmacopeia of the United States of America, Mack Printing: Easton, 1942, p. 373.

17. Forti, J. C.; Rocha, R. S.; Lanza, M. R. V.; Bertazzoli, R.; J. Electroanal. Chem. 2007, 601, 63 .

18. Forti, J. C.; Nunes, J. A.; Lanza, M. R. V.; Bertazzoli, R.; J. Appl. Electrochem. 2007, 37, 527.

19. Solomons, T. W. G.; Química Orgânica, $8^{\mathrm{a}}$ ed., Livros Técnicos e Científicos: Rio de Janeiro, 2006, vol. 2. 